\title{
Zeros dos Polinômios Característicos dos Métodos BDF
}

M. MENEGUETTE Jr. ${ }^{1}$, V.A. BOTTA², Departamento de Matemática, Estatística e Computação, FCT, UNESP, 19060-900 Presidente Prudente, SP, Brasil.

Resumo. O estudo da estabilidade de métodos numéricos possui um grande potencial em pesquisa. Como a análise da estabilidade está relacionada aos zeros do polinômio característico do método, é importante determinar o comportamento de tais zeros. Através de testes numéricos é possível verificar facilmente que os zeros dos polinômios característicos dos métodos BDF são distintos (para $K$ fixo). Mas a prova da validade deste resultado para toda a família dos métodos, nunca feita anteriormente, será apresentada neste trabalho com o uso das order stars, que são conjuntos que definem uma partição no plano complexo.

\section{Introdução}

Os métodos numéricos para a solução de equações diferenciais ordinárias fazem parte de uma área em crescente expansão, principalmente os métodos multiderivadas de passo múltiplo, que foram originalmente introduzidos com o intuito de obter métodos com menor número de passos e melhor precisão. Por possuírem boas propriedades de estabilidade, como grandes regiões de estabilidade absoluta, são indicados para resolver problemas stiff, que dizem respeito a problemas com repentinas mudanças de dinâmica. Dentre os mais conhecidos e utilizados em tais problemas destacam-se os métodos BDF (Backward Differentiation Formulae), representados por

$$
\sum_{j=0}^{K} \alpha_{j} y_{n+j}=h \beta_{1} f_{n+K},
$$

onde $\alpha_{j}(j=0, \ldots, K)$ e $\beta_{1}$ são constantes reais e $h$ é o tamanho do passo.

Associados aos métodos BDF existem dois polinômios característicos, que são

$$
\rho(z)=\sum_{j=0}^{K} \alpha_{j} z^{j} \text { e } \sigma(z)=\beta_{1} z^{K} .
$$

O comportamento das raízes de $\rho(z)=0$ é um item muito importante na análise da estabilidade de métodos numéricos, pois um método é zero-estável quando

\footnotetext{
${ }^{1}$ messias@fct.unesp.br

${ }^{2}$ botta@fct.unesp.br
} 
$\rho(z)=0$ tem todas as suas raízes no disco unitário e as raízes de módulo um são simples. Além disso, a zero-estabilidade e a consistência (ordem $p \geq 1$ ) são condições necessárias e suficientes para a convergência do método. Portanto, a análise da estabilidade é de fundamental importância no contexto dos métodos numéricos.

Testes numéricos revelam, para $K$ fixo, que os zeros dos polinômios característicos associados aos métodos BDF são distintos. Uma demonstração algébrica deste resultado não existe na literatura e então, neste trabalho, será apresentada a prova deste resultado para toda a família dos métodos através da teoria das order stars.

\section{Order Stars}

A teoria das order stars tem como objetivo principal explorar diferentes características de algoritmos numéricos para a solução de equações diferenciais como propriedades de funções analíticas em várias regiões do plano complexo, isto é, descrever ordem, estabilidade e suas relações como características de certas funções complexas.

Foi desenvolvida por Wanner, Hairer e Norsett [6] e é muito útil na análise de famílias de métodos e respectivas barreiras a que elas estão sujeitas principalmente em relação à estabilidade.

Conforme Iserles e Norsett [2], a função geradora das order stars para os métodos BDF é dada por

$$
S(z)=\frac{\sigma\left(e^{z}\right)}{\rho\left(e^{z}\right)}-\frac{1}{z}, z \in C .
$$

A função $S(z)$ define uma partição no plano complexo através dos conjuntos

$$
\begin{aligned}
& A_{+}=\{z \in C: \operatorname{Re}(S(z))>0\} \\
& A_{-}=\{z \in C: \operatorname{Re}(S(z))<0\} \\
& A_{0}=\{z \in C: \operatorname{Re}(S(z))=0\}
\end{aligned}
$$

sendo $A_{+}$e $A_{-}$chamados de order star e order star dual, respectivamente.

Um estudo mais detalhado sobre as order stars para os métodos BDF pode ser encontrado em [1].

A seguir são apresentados alguns resultados importantes na teoria das order stars, conforme [3].

Definição 2.1. $O$ índice $\iota(z)$ de um ponto $z \in A_{0}$ é definido como sendo o número de setores de $A_{-}$adjacente $a z$.

Seja $z \in A_{0}$ e $p=\iota(z)>0$. Se $S(z)$ é analítica em $z$ e o ponto é aproximado por precisamente $p$ setores de $A_{-}$, cada um com ângulos de $\frac{\pi}{p}$, dizemos que $z$ é regular.

Proposição 2.1. Seja $z_{0}$ um pólo de $S(z)$ de multiplicidade $m$. Então $\iota\left(z_{0}\right)=m$ e $z_{0}$ é regular.

O resultado a seguir estabelece a zero-estabilidade do método numérico através das order stars. 
Proposição 2.2. O método é zero-estável se, e somente se, os pólos de $S(z)$ estão no semi-plano esquerdo fechado e os pólos ao longo do eixo imaginário são simples.

Convém lembrar que para demonstrar esta proposição é preciso utilizar a transformação $z \rightarrow \ln z$, que mapeia o interior do disco unitário no semi-plano $\operatorname{Re}(z)<0$ e o círculo unitário no eixo imaginário.

O próximo resultado relaciona a ordem do método numérico com a quantidade de setores das regiões $A_{+}$e $A_{-}$.

Proposição 2.3. Se o método numérico tem ordem $p$, então $p-1$ setores de $A_{+}$e $p-1$ setores de $A_{-}$aproximam-se da origem com ângulos assintóticos de $\frac{\pi}{p-1}$ cada.

Proposição 2.4. Se o método numérico tem ordem $p \geq 2$ então $\iota(0)=p-1$ e a origem é um ponto regular da order star.

São utilizadas as nomenclaturas $A_{+}$-regiões e $A_{-}$-regiões para representar as componentes relacionadas à $A_{+}$e $A_{-}$, respectivamente. Além disso, a curva $\gamma$ é chamada de loop se é uma curva simples, fechada e limitada em $A_{0}$.

Se $S(z)=0$ segue que $z \in A_{0}$. Então, os zeros e os pólos de $S(z)$ encontram-se em $A_{0}$. Os dois resultados apresentados a seguir determinam a posição relativa desses pontos.

Sejam os conjuntos

$$
\begin{aligned}
J & =\{z \in C:|\operatorname{Im}(z)| \leq \pi\} \\
J^{+} & =\{z \in J: \operatorname{Re}(z)>0\} \\
J^{-} & =\{z \in J: \operatorname{Re}(z)<0\} .
\end{aligned}
$$

Proposição 2.5. Seja $\gamma$ um loop tal que $\gamma \cap \partial J=\emptyset$ e $\gamma \cap J \neq \emptyset$. Então existe sobre $\gamma$ exatamente um pólo de $S(z)$ entre duas raizes de $S(z)=0$. Além disso, se $z_{0} \in$ int $J$ é um pólo de $S(z)$ de multiplicidade $m$, então é aproximado por $m$ setores de $A_{+}$e $m$ setores de $A_{-}$com ângulos de $\frac{\pi}{m}$ cada.

Proposição 2.6. Seja $F$ a região limitada por $A_{+}$ou a região limitada por $A_{-}$tal que $\{\mathbb{R}+i \pi\} \cap \mathrm{clF} \neq \emptyset$ e sejam

$$
\begin{aligned}
& x_{-}=\min \{x \in \mathbb{R}: x+i \pi \in c l F\}>-\infty \\
& x_{+}=\max \{x \in \mathbb{R}: x+i \pi \in c l F\}<\infty .
\end{aligned}
$$

Seja $z_{0} \in \partial F \cap$ intJ uma raiz de $S(z)=0$. Então

1. se $F$ é uma $A_{-}$-região então ou $x_{-}+i \pi$ é um pólo de $S(z)$ ou existe um pólo de $S(z)$ ao longo da porção orientada positivamente de $\partial F$ de $x_{-}+i \pi$ à $z_{0}$;

2. se $F$ é uma $A_{+}$-região então ou $x_{+}+i \pi$ é um pólo de $S(z)$ ou existe um pólo de $S(z)$ ao longo da porção orientada positivamente de $\partial F$ de $x_{+}+i \pi$ à $z_{0}$.

Resultados análogos são válidos considerando $\mathbb{R}-i \pi$ no lugar de $\mathbb{R}+i \pi$. 
As demonstrações dos dois resultados anteriores encontram-se em [2].

As figuras a seguir representam as order stars para os métodos BDF em $\operatorname{Re}(z) \in[-2,2]$ e $\operatorname{Im}(z) \in[-\pi, \pi]$.

A área escura representa as $A_{+}$-regiões e a área complementar representa as $A_{-}$-regiões. Os pontos que encontram-se em $A_{0}$ são os pólos de $S(z)$ e o ponto na origem de cada figura representa a raiz principal de $\rho(z)=0$, que é a raiz 1 de $\rho(z)=0$.

Pode-se observar que cada figura possui $p-1$ setores, ou seja, $K-1$ setores, pois no caso dos métodos BDF a ordem é dada por $p=K$, como pode ser visto em [5]. Além disso, os métodos BDF são zero-estáveis para $K \leq 6$, o que pode ser verificado através da Proposição 2.2.

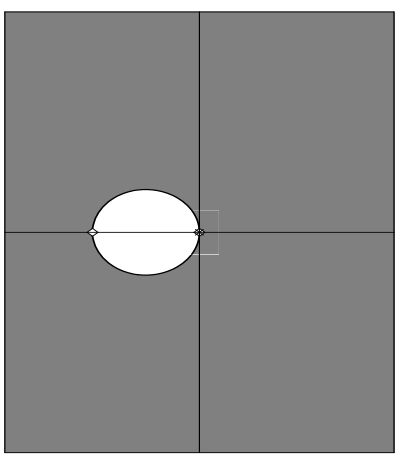

Figura 1: $K=2$

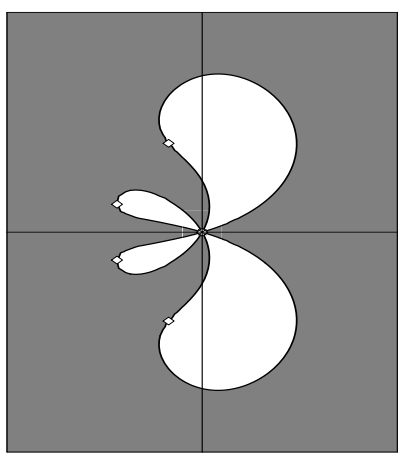

Figura $4: K=5$

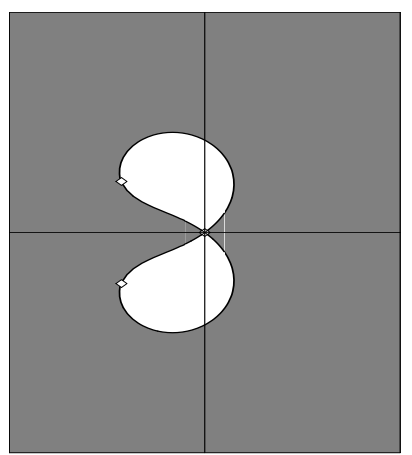

Figura 2: $K=3$

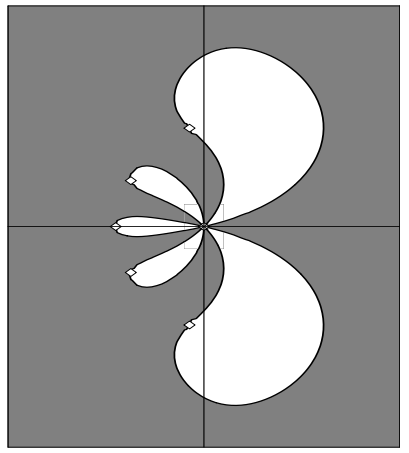

Figura 5: $K=6$

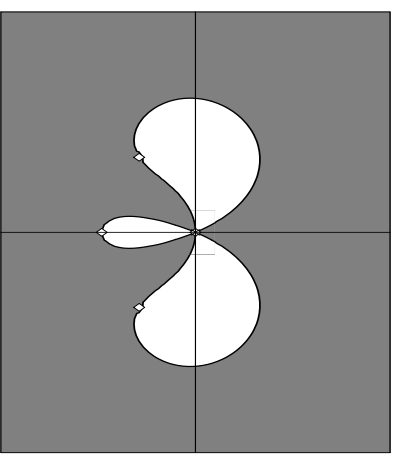

Figura 3: $K=4$

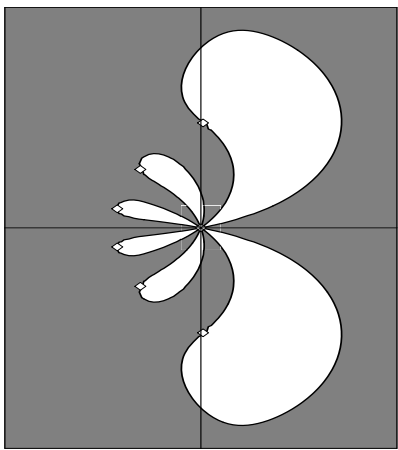

Figura $6: K=7$ 


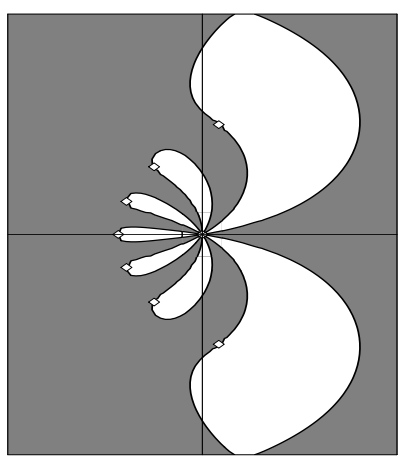

Figura $7: K=8$

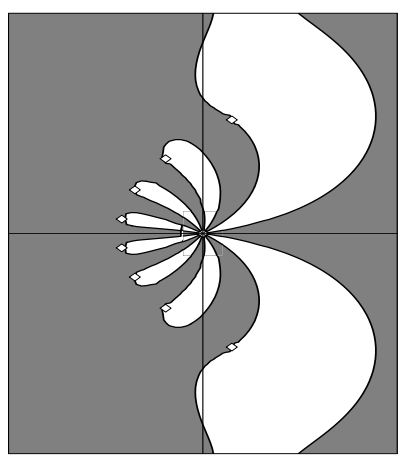

Figura 8: $K=9$

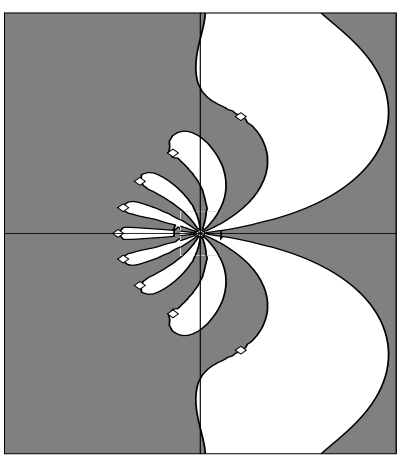

Figura 9: $K=10$

A seguir é apresentado o resultado principal deste trabalho.

Proposição 2.7. Os zeros dos polinômios característicos associados aos métodos BDF são distintos.

Demonstração. Observe primeiramente que $z_{0}=\infty$ é um pólo de ordem 0 de $S(z)$, pois $\xi=0$ é um pólo de ordem 0 de $S\left(\frac{1}{\xi}\right)$.

Portanto, da Proposição 2.1 segue que $\iota(\infty)=0$.

Logo, as order stars para os métodos BDF possuem $K-1$ setores de $A_{-}$que aproximam-se da origem, sendo tais setores limitados, visto que $\iota(\infty)=0$, ou seja, nenhum setor de $A_{-}$é ilimitado.

Portanto, as order stars para os métodos BDF possuem $K-1$ loops.

Além disso, $z_{0}=0$ é uma raiz de $S(z)=0$.

É preciso analisar dois casos:

1. $\gamma \cap \partial J=\emptyset$ e $\gamma \cap J \neq \emptyset$ :

Da Proposição 2.5 segue que existe exatamente um pólo de $S(z)$ entre duas raízes de $S(z)=0$. Como $z_{0}=0$ é raiz de $S(z)=0$ então cada loop possui um pólo de $S(z)$.

As figuras 1, 2, 3, 4, 5 e 6 representam este caso.

2. $\{\mathbb{R}+i \pi\} \cap c l F \neq \emptyset$, onde $F$ pode ser uma $A_{+}$-região ou $A_{-}$-região:

Da Proposição 2.6 segue que existe um pólo de $S$ sobre o loop $\gamma$ tal que $\gamma \cap \partial J \neq \emptyset$, conforme pode ser visto através das figuras 7,8 e 9 .

Portanto, de ambos os casos, desde que a origem é uma raiz de $S(z)=0$, então cada loop possui um pólo de $S(z)$, ou seja, uma raiz de $\rho(z)=0$.

Como já foi citado anteriormente, as order stars para os métodos BDF possuem $K-1$ loops.

Como cada setor possui uma raiz de $\rho(z)=0$, pode-se concluir que $\rho(z)=0$ possui $K$ raízes distintas, já que $K-1$ raízes encontram-se nos loops das order stars e a outra raiz é $z_{0}=1$ (representada na origem de cada figura), que é a raiz principal de $\rho(z)=0$. Portanto, $\rho(z)=0$ possui $K$ raízes distintas. 


\section{Conclusão}

Uma demonstração algébrica deste comportamento dos zeros dos polinômios característicos associados aos métodos BDF não existe na literatura. Além disso, o uso das order stars para demonstrar resultados deste tipo é ainda uma técnica pouco utilizada. Portanto, a demonstração apresentada neste trabalho poderá ser útil na análise de outros resultados, abrindo novos caminhos para a pesquisa de questões relacionadas a métodos numéricos.

Outro fato importante a ser destacado é que os métodos BDF compõem, para $L=1$, a família dos métodos $(K, L)$ de Brown, cujo estudo detalhado pode ser encontrado em [5]. Então, esta técnica será útil para mostrar também que os zeros dos polinômios característicos associados aos métodos $(K, L)$ de Brown, para qualquer valor de $L$, são distintos. Portanto, será determinado o comportamento dos zeros dos polinômios característicos de uma grande classe de métodos numéricos, o que representa um significativo avanço nas questões de estabilidade de métodos numéricos, pois o fato dos zeros serem distintos indica um novo caminho a ser perseguido no sentido de provar uma conjectura postulada por Jeltsch [4] de que todo método $(K, L)$ zero-estável é também $A_{0}$-estável.

\footnotetext{
Abstract. The study of the stability of numerical methods has a great potential in research. As the analysis of the stability is related to the zeros of the characteristic polynomial of the method, it is important to determine the behavior of such zeros. Through numerical tests it is possible to verify easily that the zeros of the polynomial characteristic of the BDF methods are differents (for $K$ fixed). But the proof of the validity of this result for all family of the methods, never done previously, it will be presented in this work with the use of the order stars, that are sets that define one partition in the complex plan.
}

\section{Referências}

[1] V.A. Botta, "Zeros de Polinômios Característicos e Estabilidade de Métodos Numéricos", Monografia de Qualificação de Doutorado, ICMC - USP, São Carlos, 2005.

[2] A. Iserles, S.P. Nørsett, A proof of the first Dahlquist barrier by order stars, BIT, 24 (1984), 529-537.

[3] A. Iserles, S.P. Nørsett, "Order Stars", Chapman and Hall, London, 1991.

[4] R. Jeltsch, $A_{0}$-stability and stiff stability of Brown's multistep multiderivative methods, Numer. Math. 32 (1979), 167-181.

[5] M. Meneguette Jr., "Multistep Multiderivative Methods and Related Topics", Tese de Doutorado, Oxford, UK, 1987.

[6] G. Wanner, E. Hairer, S.P. Nørsett, Order stars and stability theorems, BIT, 18 (1978), 475-489. 